\title{
Video Article \\ Functional and Physiological Methods of Evaluating Median Nerve Regeneration in the Rat
}

\author{
Diogo Casal ${ }^{\star^{1,2}}$, Eduarda Mota-Silva ${ }^{\star^{3}}$, Inês Iria ${ }^{4,5}$, Diogo Pais ${ }^{1}$, Ana Farinho ${ }^{6}$, Sara Alves ${ }^{7}$, Cláudia Pen $^{7}$, Luís Mascarenhas-Lemos ${ }^{7}$, \\ José Ferreira-Silva ${ }^{7}$, Mário Ferraz-Oliveira ${ }^{7}$, Valentina Vassilenko ${ }^{3}$, Paula A. Videira ${ }^{4,5}$, João Goyri-O'Neill ${ }^{1}$ \\ ${ }^{1}$ Anatomy Department, NOVA Medical School \\ ${ }^{2}$ Plastic and Reconstructive Surgery Department and Burn Unit, Centro Hospitalar de Lisboa Central \\ ${ }^{3}$ Physics Department, Faculty of Sciences and Technology, LIBPhys \\ ${ }^{4}$ Glycoimmunology, CEDOC, NOVA Medical School \\ ${ }^{5}$ UCIBIO, Life Sciences Department, Faculty of Sciences and Technology, Universidade NOVA de Lisboa \\ ${ }^{6}$ Tissue Repair Unit, CEDOC, NOVA Medical School \\ ${ }^{7}$ Pathology Department, Centro Hospitalar de Lisboa Central \\ *These authors contributed equally
}

Correspondence to: Diogo Casal at diogo_bogalhao@yahoo.co.uk

URL: https://www.jove.com/video/59767

DOI: doi:10.3791/59767

Keywords: Medicine, Issue 158, median nerve, nerve regeneration, peripheral nervous system, repair, rat, experimental model, surgery, functional tests

Date Published: 4/18/2020

Citation: Casal, D., Mota-Silva, E., Iria, I., Pais, D., Farinho, A., Alves, S., Pen, C., Mascarenhas-Lemos, L., Ferreira-Silva, J., Ferraz-Oliveira, M., Vassilenko, V., Videira, P.A., Goyri-O'Neill, J. Functional and Physiological Methods of Evaluating Median Nerve Regeneration in the Rat. J. Vis. Exp. (158), e59767, doi:10.3791/59767 (2020).

\section{Abstract}

The main goal of this investigation is to show how to create and repair different types of median nerve (MN) lesions in the rat. Moreover, different methods of simulating postoperative physiotherapy are presented. Multiple standardized strategies are used to assess motor and sensory recovery using an $\mathrm{MN}$ model of peripheral nerve lesion and repair, thus permitting easy comparison of the results. Several options are included for providing a postoperative physiotherapy-like environment to rats that have undergone MN injuries. Finally, the paper provides a method to evaluate the recovery of the MN using several noninvasive tests (i.e., grasping test, pin prick test, ladder rung walking test, rope climbing test, and walking track analysis), and physiological measurements (infrared thermography, electroneuromyography, flexion strength evaluation, and flexor carpi radialis muscle weight determination). Hence, this model seems particularly appropriate to replicate a clinical scenario, facilitating extrapolation of results to the human species.

Although the sciatic nerve is the most studied nerve in peripheral nerve research, analysis of the rat MN presents various advantages. For example, there is a reduced incidence of joint contractures and automutilation of the affected limb in MN lesion studies. Furthermore, the MN is not covered by muscle masses, making its dissection easier than that of the sciatic nerve. In addition, MN recovery is observed sooner, because the $\mathrm{MN}$ is shorter than the sciatic nerve. Also, the $\mathrm{MN}$ has a parallel path to the ulnar nerve in the arm. Hence, the ulnar nerve can be easily used as the nerve graft for repairing $\mathrm{MN}$ injuries. Finally, the $\mathrm{MN}$ in rats is located in the forelimb, akin to the human upper limb; in humans, the upper limb is the site of most peripheral nerve lesions.

\section{Video Link}

The video component of this article can be found at https://www.jove.com/video/59767/

\section{Introduction}

Peripheral nerve lesions occur regularly as a result of trauma, infection, vasculitis, autoimmunity, malignancy, and/or radiotherapy ${ }^{1,2}$.

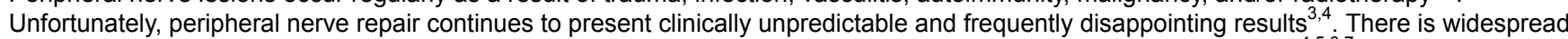
consensus that considerable basic and translational research is still needed to improve the prospect of those affected ${ }^{4,5,6,7}$.

The rat MN shows great similarities to that of humans ${ }^{8,9}$ (Figure 1). Originating from the brachial plexus in the axillary region, this nerve descends into the medial aspect of the arm, reaching the elbow, and branching off to the majority of the muscles in the ventral compartment of the forearm. The MN reaches the hand, where it innervates the thenar muscles and the first two lumbrical muscles as well as to part of the rat's hand $\operatorname{skin}^{9}$ (Figure 1).

Using the rat $\mathrm{MN}$, it is possible to adequately replicate peripheral nerve lesions in humans ${ }^{10,11,12}$. This nerve has several potential research advantages relative to the customarily used sciatic nerve. Because the $\mathrm{MN}$ is located in the forelimb of rats (akin to the human upper limbs), it can be damaged experimentally with a much smaller impact on the rat's wellbeing, compared to the sciatic nerve, which innervates a 
substantial portion of the pelvic limb ${ }^{13}$. Additionally, in humans most clinical lesions occur in the upper limb, which corresponds to the rat's forelimb ${ }^{10,11,12,14,15,16}$.

This paper shows how to produce different types of $\mathrm{MN}$ lesions in the rat. Moreover, different ways to simulate postoperative physiotherapy are presented. Finally, tests to evaluate functional recovery of the $\mathrm{MN}$ are described. There are multiple standardized strategies available to assess motor and sensory recovery using an MN model of peripheral nerve lesion and repair, thus permitting an easy comparison of results. The MN model is particularly suitable to replicate the clinical scenario, facilitating extrapolation of results to the human species.

\section{Protocol}

All procedures involving animal subjects were approved by the Institutional Animal Care and Use Committee and Ethical Committee at Nova University Medical School, Lisbon, Portugal (08/2012/CEFCM).

\section{Median nerve surgery}

NOTE: Follow aseptic technique during the surgery. Use personal protective instruments (PPE) and wear a sterile surgical gown ${ }^{17}$. Autoclave all the required surgical instruments prior to the surgery (see the Table of Materials).

1. Use 12-week-old Wistar rats. Provide them with ad libitum food and water with $12 \mathrm{~h}$ light-dark cycles 7 days prior to surgery. Prior to anesthesia, weigh the rat to determine the amount of anesthetic required.

2. Anesthetize the rat with an intraperitoneal injection of a mixture of ketamine (40-80 mg/kg body weight) and xylazine (5-10 mg/kg of body weight). Check for the depth of anesthesia by the lack of response to a toe pinch and by observing of respiration rate throughout the entire procedure ${ }^{18,19}$. Provide additional analgesia if a respiratory rate over 110 cycles/min or motor response to toe pinching is observed ${ }^{18,20}$.

3. Inject $1 \mathrm{mg} / \mathrm{kg}$ of meloxicam subcutaneously to provide preemptive analgesia ${ }^{20,21}$

4. To avoid corneal abrasion during the surgery, apply ophthalmic gel to both the eyes.

5. Use a depilatory cream to remove the hair over the medial aspect on the right. Once done, wash with warm saline to remove the cream ${ }^{17}$.

6. Lay the rat in supine position on a heating pad. Apply an iodine or chlorhexidine-based surgical scrub over the operative site. Leave it for at least $15 \mathrm{~s}$ and then wipe with ethanol. Repeat the application 3x. Ensure that the scrub is in contact with the skin for at least 2 min prior to proceeding with the surgery.

NOTE: Contact the infection control authority at your research unit for alternative protocols to prevent surgical site infection ${ }^{19}$.

7. Drape the surgical area ${ }^{17}$. NOTE: Perform all procedures under strict aseptic conditions ${ }^{19}$.

8. Incise the skin in the medial aspect of the right arm and pectoral region to the deep fascial plane, using a number 15 scalpel blade. Carefully cauterize any bleeding vessels using an electric cautery.

9. Carefully divide the brachial fascia, which presents as a whitish sheath covering the muscles, using a thermocautery or a pair of blunt scissors, taking care not to damage the vascular and nerve structures in the medial aspect of the arm.

10. Open a pair of scissors bluntly beneath the terminal insertion of the pectoralis major muscle, in order to tease this muscle away from the underlying axillary artery and vein, as well as from the terminal branches of the brachial plexus.

11. Divide the insertion of the pectoralis major muscle with an electric cautery. Expose and section the pectoralis minor muscle.

12. Bluntly dissect the MN from the brachial vessels and from the ulnar nerve starting from the axillary region up to the elbow. This allows exposure of the different terminal branches of the brachial plexus, namely the median, the ulnar, the radial, the axillary and the musculocutaneous nerves (Figure 2).

13. Separate different experimental groups as described below.

1. Create the Sham group by only dissecting the MN.

2. Create the Crush group by compressing the $\mathrm{MN}$ in the middle portion of the arm for $15 \mathrm{~s}$ using a number 5 microsurgery forceps, or a similar instrument ${ }^{22,23}$.

3. Create the Excision group using a pair of microsurgery scissors to excise a $10 \mathrm{~mm}$-long segment from the central part of the MN in the arm. Ligate the proximal stump of the nerve with an 8/0 Nylon suture to prevent axonal growth.

4. Create the Graft group using the $10 \mathrm{~mm}$-long-segment of the $\mathrm{MN}$ described in the latter step and rotating it $180^{\circ}$. Suture the proximal and distal stumps of the sectioned $\mathrm{MN}$ to the nerve graft using interrupted 10/0 Nylon stitches.

14. Close the skin wound using interrupted $5 / 0$ Nylon stitches ${ }^{10,24}$.

15. Provide postoperative analgesia with $7 \mathrm{~mL}$ of cherry-flavored acetaminophen mixed with $43 \mathrm{~mL}$ of tap water ${ }^{25}$ to obtain a concentration of $4.48 \mathrm{mg} / \mathrm{mL}$ in $50 \mathrm{~mL}$ plastic water bottles made available to the rats ad libitum for 3 days ${ }^{25}$.

\section{Housing and physiotherapy}

1. Allow rats to have contact with the physiotherapy apparatuses $2-4$ weeks before surgery, in order to ensure an easier and faster adaptation to the exercise settings. Perform the exercises by following the procedures detailed below.

2. Once a day, place each rat inside an individual physiotherapy sphere, and subsequently place the sphere in a room with few obstacles. Let the rat ambulate and explore the room freely for half an hour.

3. House the rats individually in solitary cages with incorporated running wheels to help them exercise.

4. Form groups of 4-5 animals and house these groups in personalized cages. Personalize the cages by including ladders, ropes, running wheels, and other environmental enriching elements.

5. Return individual rats to personalized cages the day after surgery.

6. Resume physiotherapy exercises 3 days after surgery. 


\section{Functional tests}

1. One week before starting to perform the functional tests, familiarize the rats with the food treats to be used as positive reinforcement. Provide this reinforcement after the successful completion of each test, before and after surgery. After the initial training period of 3 weeks, resume all tests 1 week after surgery.

2. Conduct the tests in the evening, when the rats are naturally more active. Resume tests 1 week after surgery.

3. Perform the Grasping Test by placing the rat on a grid and lift it by its tail, letting it grab the grid with its forepaws ${ }^{11,26}$. Assign a "Positive" score if the rat can grab the grid with both forepaws. Assign a "Negative" score if the rat cannot grab the grid with the injured paw. NOTE: A positive Grasping Test indicates that the motor component of the MN is functioning ${ }^{16,27}$.

4. Perform a Pin Prick Test ${ }^{28,29}$.

1. Make a plastic platform with a $4 \mathrm{~mm} \times 4 \mathrm{~mm}$ square grid pattern. Provide support to this grid with a $21 \mathrm{~cm}$ long metallic frame.

2. Place the rat on the platform and cover the grid with a $15.5 \mathrm{~cm} \times 15.5 \mathrm{~cm} \times 11 \mathrm{~cm}$ transparent plastic box. Wait for few minutes until normal activities (e.g., exploratory and major grooming) subside.

3. Start the test when the rat is stationary and standing on its four paws.

4. With the help of a mirror, insert an aesthesiometer (e.g., number 4 Von Frey hair with a bending force of $25 \mathrm{~g}$ ) through the mesh and poke the palmar aspect of the forepaw in the skin territory of the MN (Figure 1). Repeat the assessment $5 \mathrm{x}$ on each forepaw alternatively, waiting a few seconds after each evaluation.

5. Check for the bending of the Von Frey filament ${ }^{30}$ for the correct evaluation. Score the withdrawal responses as follows: "0" for no withdrawal response, "1" if the rat slowly removes the paw from the filament, "2" if the rat quickly responds to the stimulus and removes the paw or licks the paw.

NOTE: If ambulation and biting of the filament are observed repeat the stimulus, because these are considered ambiguous responses.

5. Training sessions

NOTE: Train the rats daily for 3 weeks before performing the surgery in the evening in a low-light environment. The training sessions are recommended specially for the rope climbing, ladder rung, and walking tracks tests. These can be done in the order presented before, starting with the rope climbing test, ladder rung, and finally walking tracks test. Allow the same animal a few minutes of rest before a new test.

1. During the first week, place the rat on the last third of the ladder/rope/corridor, close to the box's entrance. Condition the animal to move towards the box opening by gently touching and/or pulling the tip of its tail. Provide the rat a food treat once it enters the box, allowing it some seconds of rest before repeating the test. Repeat it $5 x$ each day for 5 days.

2. During the second week, place the animal on the second third of the ladder/rope/corridor Repeat the steps in 3.5.1.

3. During the third week, place the rat on the bottom of the ladder/rope/corridor, on the opposite side of box's entrance. Repeat the steps in 3.5.1, but only reward the animal when it completes the test correctly.

6. Perform the Ladder Rung Test.

NOTE: This test is used for assessing forelimb strength, stepping, placing, and coordination ${ }^{31}$

1. Place the rat at the bottom of the ladder $(120 \mathrm{~cm} \times 9 \mathrm{~cm} \times 2 \mathrm{~cm}$ with 18 steps $1.5 \mathrm{~cm}$-thick, spaced $4 \mathrm{~cm}$ apart) and gently touch the rat's tail. Ensure that the ladder is placed at an inclination of $10^{\circ}$ and leads to a $13.20 \mathrm{~cm} \times 11 \mathrm{~cm}$ opening on a dark wooden $31.5 \mathrm{~cm} \times$ $35 \mathrm{~cm} \times 35 \mathrm{~cm}$ box.

2. Start the timer once the rat starts climbing the ladder and stop the timer once the rat's snout crosses the box's entrance.

3. Record the time and repeat the test $3 x$, each separated at least by a 1 min interval.

7. Rope Climbing

NOTE: This test is used for assessing grasping strength, which is dependent on MN recovery ${ }^{32}$

1. Put the rat at the bottom of the rope and persuade it to climb by gently touching its tail. Start the timer once the animal starts climbing and stop it the moment the rats' snout crosses the platform's entrance.

2. For each test, record the time taken to climb to the platform and the number of slips of the injured paw while the rat climbs up the rope. Consider the test valid if the animal does not hesitate during the task or does not stop climbing. Provide the rat with a snack after performing the task correctly.

3. Record the time and repeat the test $3 x$, each separated at least by a 1 minute interval.

8. Walking Tracks

NOTE: This test is used for the evaluation of forelimb motor recovery ${ }^{33,34}$

1. Set up an apparatus consisting of a confined walkway $16.5 \mathrm{~cm}$ in height $\times 8.7 \mathrm{~cm}$ in width $\times 43 \mathrm{~cm}$ in length. Ensure that this leads to a rectangular $8.8 \mathrm{~cm} \times 8.2 \mathrm{~cm}$ opening in one of the walls of a black wooden $23 \mathrm{~cm} \times 36 \mathrm{~cm} \times 28 \mathrm{~cm}$ box. Include a vertical sliding door to close the box's entrance rapidly. Include a removable top to be used for retrieving the rat $^{33,34}$.

2. Place a piece of graph paper on the corridor's floor. Grab the rat by its tail and let it hold a painting brush soaked in methylene blue. Position the rat at the entrance of the corridor to make it walk inside the box. Remove the graph paper from the corridor's floor and repeat the test until a good representative impression of both forepaws is obtained.

3. From the obtained prints, select one with clear successive forepaw prints, photograph them in a tiff or jpeg format, and measure the following parameters using the open access software $\mathrm{FIJI}^{35}$.

NOTE: First, calibrate each image using the markings in the graph paper (Analyze | Set scale) Second, convert each image to an 8bit format (Image | Type | 8-bit). Subsequently, use the Rectangular Selection Tool to select the paw print. Crop this portion of the image (Image | Crop). In each image, highlight the pawprints and remove the background by thresholding the image (Image | Adjust | Threshold).

1. Measure the stance factor by measuring the paw impression area. Use the Rectangular Selection Tool to select the paw print and press Control $+\mathbf{M}$.

2. Measure the print length factor by measuring the longest length of the paw impression (for steps 3.8.3.2-3.8.3.6, use the

Straight-Line Selection Tool for selecting the two most distant points and press Control $+\mathbf{M})$.

3. Measure the finger spread factor by measuring the widest width of the paw impression. 
4. Measure the intermediate finger spread factor by measuring the widest width between the second and third fingers.

5. Measure the stride length by measuring the distance between homologous points of successive paw impressions on a given side.

6. Measure the base of the support by measuring the perpendicular distance between the central portion of the paw impression and the direction of movement ${ }^{29,33,36}$ NOTE: Perform the last two measurements in two pairs of representative consecutive bilateral paw impressions ${ }^{33}$.

\section{Physiological measurements}

1. Infrared Thermography (IRT) ${ }^{37,38,39}$.

1. Ensure that the temperature of the room where the measurements will be performed is between $18^{\circ} \mathrm{C}-25^{\circ} \mathrm{C}$ using a normal digital hydrothermometer with a thermal resolution of $0.1^{\circ} \mathrm{C}$. Ensure that no significant heat sources (e.g., computers or refrigerators) are present.

2. Acclimate the rats by bringing them to the evaluation room $2 \mathrm{~h}$ prior to the evaluation. Before starting the experiment, anesthetize the rat as described above (steps 1.3-1.6) or following the institution's protocol. Check for the lack of the response to a toe pinch before starting the experiment.

3. Switch on the infrared thermographic camera 15 min prior to the acquisition and do not turn it off during the evaluations. Set the emissivity parameter of the camera to match that of the rat's skin $(\varepsilon=0.98)^{37,40,41}$.

4. Place the rat on its dorsum on a clean and stable surface with a polyethylene sponge. Ensure that there are no reflective materials and other possible sources of artefacts. Fix its forepaws carefully in supination with double face glue tape. Insert a digital thermometer $2 \mathrm{~cm}$ inside the rectum to monitor the rat's central temperature during all evaluations.

5. Hold the thermographic camera at a $90^{\circ}$ angle and $30 \mathrm{~cm}$ distance from the rat. Focus the camera on the whole animal's body. Obtain three infrared thermography images spaced $30 \mathrm{~s}$ apart.

6. Transfer the acquired thermograms to a computer and analyze them using analysis software. Define the temperature of the plantar surface of both forepaws using a fixed rectangular region of interest (e.g., $9 \times 11$ pixels) in the plantar territory of the $\mathrm{MN}$, for example in the center of the first metacarpal foot pad (Figure 1). Using the free FLIR Tools software, select the thermography by double clicking on it. On the left tools bar select the button "Add box measurement tool" and draw a rectangle of 9*11 pixels over the plantar area of both forepaws. While adjusting the rectangle its dimension in pixels can be confirmed. Perform it on both forepaws. On the right side of the image find the maximum, minimum, and average temperatures.

7. Over the previously drawn ROI, right click and select export. The mean, maximum, and minimal temperatures, as well as a matrix of temperatures of the ROI are then exported to a .csv document. These data can later be explored using data analysis software.

2. Electroneuromyographic (ENMG) Evaluation

1. Set up an electrical stimulator. Tape a pair of disposable acupuncture needles $(0.25 \mathrm{~mm} \times 25 \mathrm{~mm})$ with a negligible impedance $[<1 \Omega]$ ] and $25 \mathrm{~mm}$ between them to create electrodes for stimulation. Now connect the stimulator and the electrode to a data acquisition unit to take the incoming signals and convert them into digital signals that can be processed with the computer software.

2. Perform the evaluations in the same room, and always under the same controlled environmental conditions ${ }^{42,43,44}$. Pinch the forepaw to ensure that the rats are deeply anesthetized before starting data acquisition.

NOTE: Deep anesthesia is of paramount importance to minimize variability associated with spontaneous voluntary and/or involuntary movements by the rats ${ }^{43}$

3. Expose the $\mathrm{MN}$ on both sides under the surgical microscope as described in steps 1.8-1.13. Use a number 15 scalpel blade to prolong the brachial incisions into the forearms with a ventral midline incision.

4. Expose the superficial aspect of the flexor digitorum sublimis muscles by bluntly separating the overlying antebrachial fascia using iris scissors. Insert the ground needle in the quadriceps femoris muscle of the left hindlimb to connect the signal ground plug.

5. Start with the right forepaw and place the recording electrodes into the flexor digitorum sublimis muscle belly of the forepaw and the stimulation electrode proximal to the lesion site in the MN. Moisten these electrodes with saline.

6. Ensure that the software is set as follows: channel input port $1(\mathrm{CH} 1)-$ stimulator to $0-10 \mathrm{~V}$; and channel input port 2 (CH2) - EMG to $30-1,000 \mathrm{~Hz}$. Start by choosing a stimulation amplitude of $10 \mathrm{mV}$ and record the compound muscle action potentials CMAPs sample rate of $50 \mathrm{kHz}$ for a duration of $40,000 \mathrm{~ms}$. Gradually increase the stimulation amplitude in $10 \mathrm{mV}$ steps until reaching $2,000 \mathrm{mV}$. Repeat the same for the left paw ${ }^{42,43,44}$.

NOTE: The signal is amplified to $1,000 \mathrm{x}$ and filtered using a $30-1,000 \mathrm{~Hz}$ band. The stimulation output is set for a single pulse with a duration of $1 \mathrm{~ms}^{42,43,44}$.

7. Open the recorded file in the recording apparatus software.

NOTE: By default, the screen will show tow windows on top in red, the stimulator pulses, and below in blue the recorder ENMGs. Sliding the horizontal scroll bar underneath the time scale allows visualization of the full record. The two main tools, Zoom tool and $I$-Beam tool, are on the bottom right side of the panel. Using the Zoom tool, it is possible to optimize the visualization of the CMAPs and explore the graphics. To guarantee a good fitting on the visualization screen, it may be necessary to adjust the display after zooming. To do so, select Display|Autoscale Waveforms. The I-beam tool allows the selection of specific areas of the graphs and performance of the desired measurements. On top of the graphs, there are three small windows where the measurements are displayed. $\boldsymbol{P}$-P shows the average amplitude value of the selected area in volts (both in the stimulator record and in ENMGs), whereas Delta-T shows the time interval of that selection.

8. Measure the parameters from the compound muscle action potential (CMPAs, described in Table 1) using the homonymous measurement tools from the software plug in "Toolbox for unsupervised classification of MUAPs and action potentials in EMG" 45

9. For each rat, determine a minimal value of stimulation voltage after which the CMAPs amplitude does not increase further. Start from a $0.05 \mathrm{mV}$ stimulus, and give successive increasing stimuli in incremental voltages of $0.05 \mathrm{mV}$.

10. Apply a stimulus $20 \%$ above this voltage in order to obtain a supramaximal stimulation value.

11. After the latter value is determined and the corresponding stimulus applied, record the next CMAPs parameters. 
3. Flexion strength evaluation

1. Use the same stimulator and stimulation electrodes to electrically stimulate the $\mathrm{MN}$ as in step 4.2. Set the input channel $\mathrm{CH} 1 \mathrm{as}$ the stimulator $(0-10 \mathrm{~V})$ and the output settings for stimuli duration of $30 \mathrm{~s}$ with pulses of $1 \mathrm{~ms}$ duration and $1 \mathrm{~Hz}$ frequency. Link a dynamometer, with a resolution of $\mathrm{d}=0.001 \mathrm{~N}$ to a computer.

NOTE: Real time visualization of data can be obtained by building a plot of force per time $(\mathrm{N} / \mathrm{s})$ using the software previously installed on the computer and linked to a dynamometer ${ }^{46}$.

2. Place the rat as described in step 4.1.4. Place a 5/0 silk suture loop through the second interosseous space of both forepaws. Attach the suture loop to the dynamometer's hook and the forepaw aligned with the dynamometer without putting excessive strain on the suture line.

3. Fix the contralateral paw with tape to avoid spurious movement interferences in the dynamometer readings.

4. Set the dynamometer to zero by clicking on the ZERO button.

5. Adjust the stimulator to a supramaximal amplitude stimulation of $1.5 \mathrm{~V}$ by adjusting the voltage knot.

6. On the PC, open the software AFH-01. Open the separator "Device" and select the device FH5. Create a new file ("Measurements1" is the name given by default) and rename the file.

7. Place the electrode on the proximal part of the $\mathrm{MN}$, click on play at the bottom of the program, record the pull on the dynamometer for $30 \mathrm{~s}$.

8. Import the values obtained to a data analysis software. Calculate the maximum and average force values and the area under the curve (AUC) for the strength $x$ time graph for each evaluation.

9. Repeat for the left forepaw.

4. Muscle weight

1. Euthanize the rats under general anesthesia by exsanguination ${ }^{47,48}$.

2. Harvest the flexor carpi radialis muscle from both forearms, dissecting the muscle from its origin until its distal tendon insertion, using a number 15 scalpel blade.

3. Weigh the muscles with a precision scale ${ }^{9,49}$.

\section{Representative Results}

A total of 34 rats were randomly divided into the following groups: Sham $(n=17)$, Excision $(n=17)$, and Nerve Graft $(n=10)$ for the operation. All rats survived surgery and the postoperative period uneventfully. One week after surgery and for the subsequent 100 days, all animals underwent the functional tests described above once a week. The representative results of each of these tests are described below.

\section{Grasping Test}

The percentage of rats with a positive response in the grasping test was highest for the Sham group. This value gradually increased over time in rats from the Crush and Nerve Graft groups (Figure 3).

\section{Pin Prick Test}

Rats from the Sham group had the best scores in the cumulative pin prick test relative to rats from the Nerve Graft group. Both had better scores than the rats in the Excision group (Figure 4).

\section{Ladder Running Test}

The rats' velocity in the ladder running test was highest in the Sham group than in the rats submitted to MN lesion. Among the latter, the time to run the ladder tended to decrease over time, paralleling MN recovery (Figure 5).

\section{Rope Test}

As in the ladder running test, the time the rats took to climb the rope was shorter in the Sham group compared to the groups in which the MN was injured. The rats' speed in this test increased when the MN was allowed to recover (Figure 6).

\section{Walking Track Analysis}

Analysis of walking tracks tended to show changes in the morphology of paw prints (Figure 7). These changes were often more pronounced in crushing injuries than in segmental nerve lesions ${ }^{5}$.

\section{Infrared Thermography}

Thermography was useful when examining temperature differences between the forepaws in the first 30 days after surgery. Temperature differences were more noticeable in rats with a more severely injured MN, such as in those from the Excision group (Figure 8 and Figure 9 ).

\section{Electroneuromyography}

Table 1 summarizes the biological importance of the electroneuromyography measurements, providing representative results for the different experimental groups. Various patterns were observed with electroneuromyography. A normal CMAP was typical of a rat from the Sham group while a polyphasic CMAP was associated with a variable degree of lesion of the MN, as in the Crush and in the Nerve Graft groups (Figure 10) In the Excision group, no CMAPs were observed.

\section{Wrist Flexion Strength}


Given that wrist flexion is mainly dependent on the MN, this test was used to evaluate motor recovery in this nerve's territory. Wrist flexion strength was closest to normal when recovery was maximal (Figure 11).

\section{Muscle Weight and Morphology}

The weight and morphology of the flexor carpis radialis muscle were dependent on MN recovery, as this muscle is innervated exclusively by the $\mathrm{MN}^{9,10}$. Thus, normal weight and morphology were observed in the Sham group. A loss of weight and muscle trophism was observed in the Crush, Nerve Graft, and Excision groups (Figure 12).

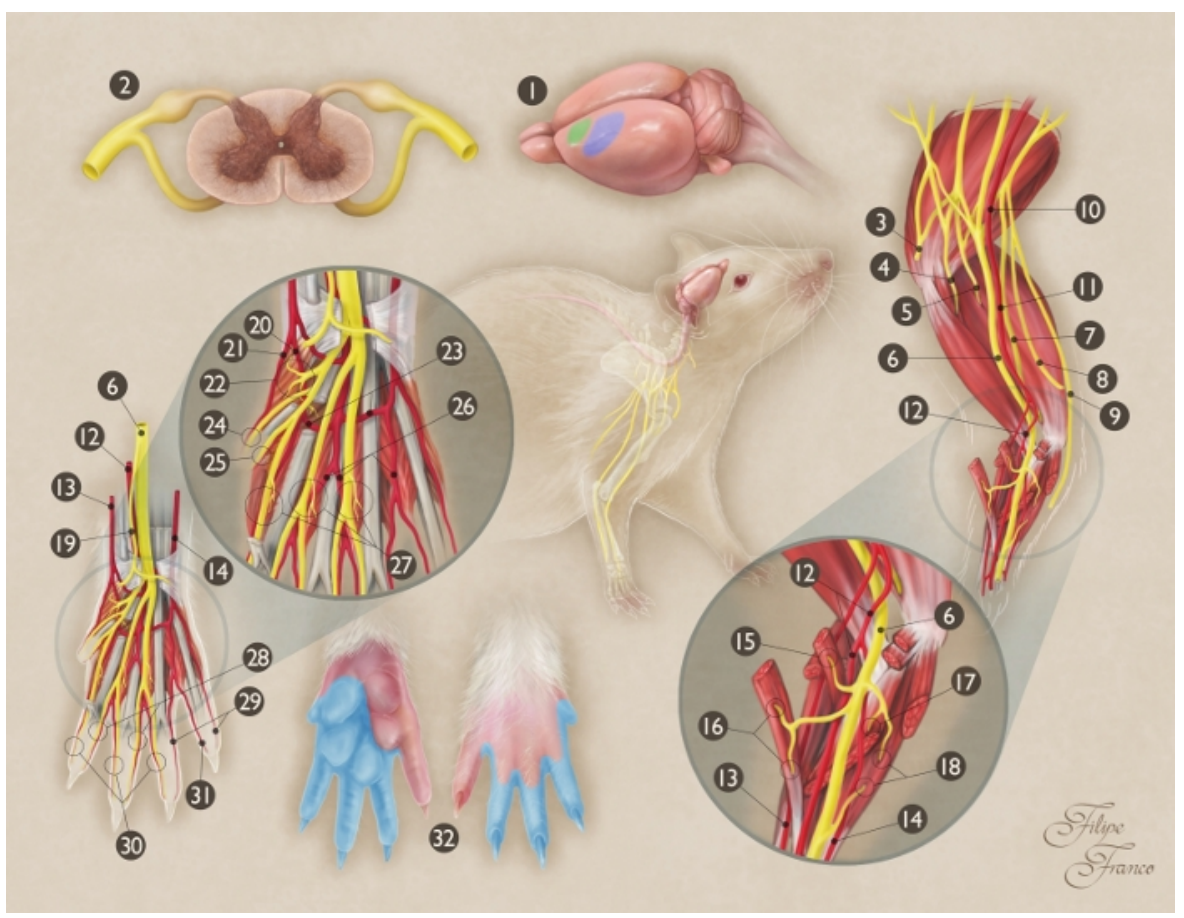

Figure 1: Schematic representation of the anatomy of the median nerve of the rat.

(1) Origin and termination of the median nerve in the rat brain (green area = primary motor area; blue area = primary sensory area). (2)

Transverse section of the spinal cord at C7 segment level; (3) Axillary nerve; (4) Musculocutaneous nerve; (5) Radial nerve; (6) Median nerve; (7) Ulnar nerve; (8) Medial cutaneous branch of the arm; (9) Medial cutaneous branch of the forearm; (10) Axillary artery; (11) Brachial artery; (12) Median artery; (13) Superficial radial artery; (14) Ulnar artery; (15) Motor branch of the median nerve to the pronator teres muscle; (16) Motor branch of the median nerve to the flexor carpis radialis muscle; (17) Motor branch of the median nerve to the flexor digitorum superficialis muscle; (18) Motor branch of the median nerve to the flexor digitorum profundus muscle; (19) Sensory branch of the median nerve to the thenar region; (20) Common palmar artery of the first interosseous space; (21) Radial palmar digital artery of the first digit; (22) Motor branch of the median nerve to the thenar muscles; (23) Palmar arterial arch; (24) Radial palmar digital nerve of the first digit; (25) Ulnar palmar digital nerve of the first digit; (26) Common palmar artery of the third interosseous space; (27) Motor branches of the terminal divisions of the median nerve to the first three lumbrical muscles; (28) Ulnar palmar digital nerves of the second, third, and fourth digits; (29) Ulnar palmar digital arteries to the fourth and fifth digits; (30) Radial palmar digital nerves of the second, third, and fourth digits; (31) Radial palmar digital artery of the fifth digit; (32) Skin territory of the median nerve in the forepaw (blue-shaded region). Please click here to view a larger version of this figure. 


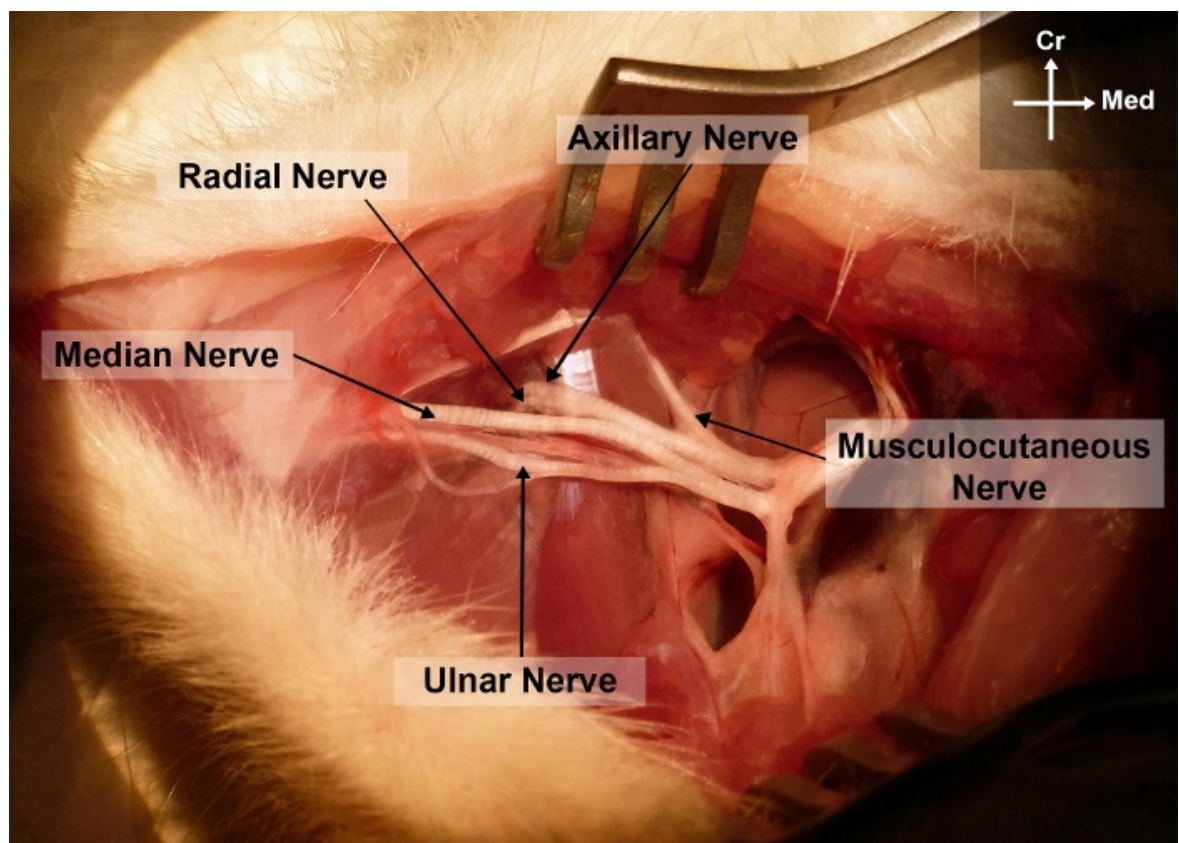

Figure 2: Photograph of the right forelimb of the rat showing the surgical anatomy of the median nerve in the arm and axillary regions. $\mathrm{Cr}$, cranial; Me, medial Please click here to view a larger version of this figure.

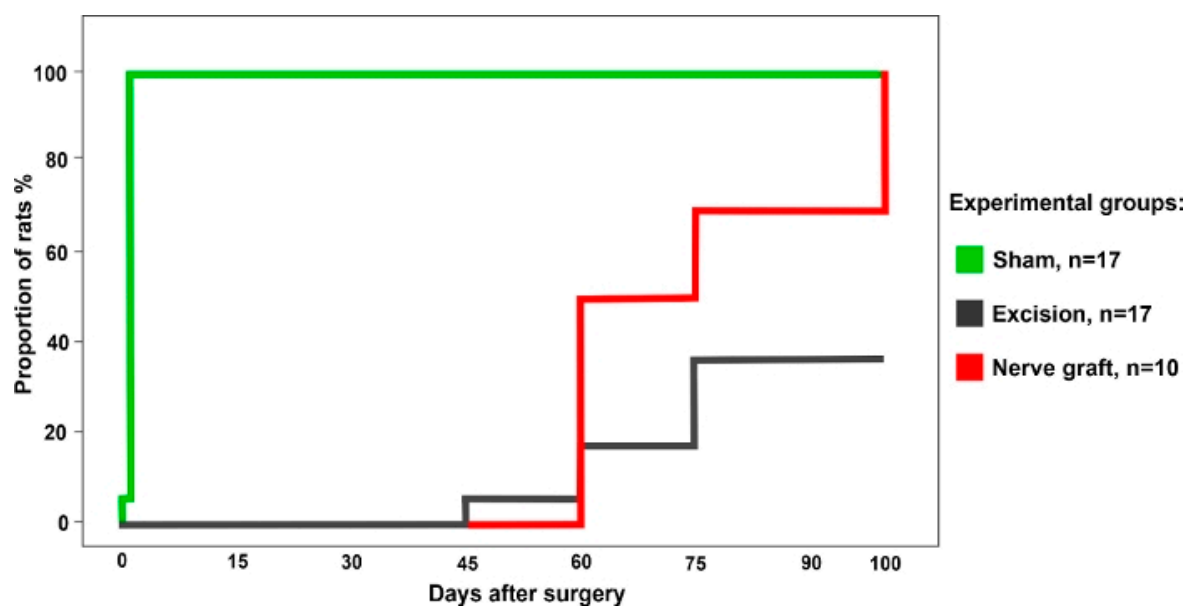

Figure 3: Percentage of rats with a positive grasping test in the different experimental group over a period of 100 days after surgery. Please click here to view a larger version of this figure. 


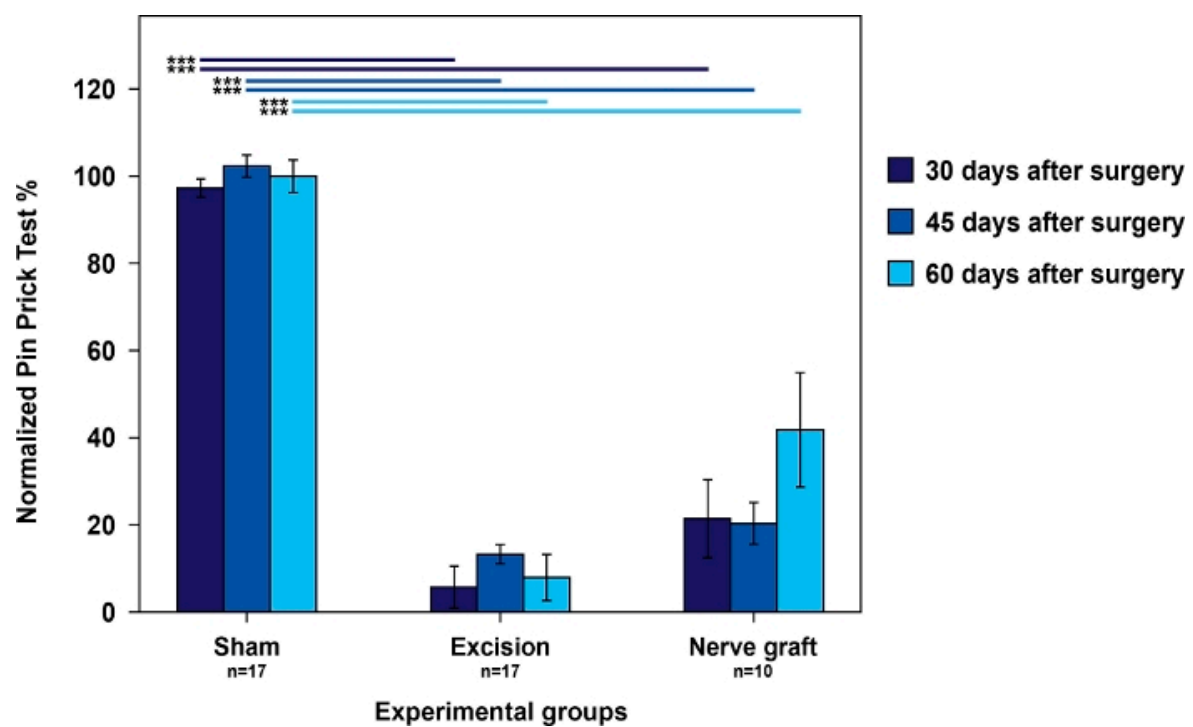

Figure 4: Nociception evaluation using cumulative pin prick test results in the operated forepaw normalized to the contralateral paw in the different experimental groups.

Vertical bars represent $95 \%$ confidence intervals. Horizontal lines in the upper part of the figure indicate statistically significant differences between experimental groups, ${ }^{* * *} p<0.001$. Please click here to view a larger version of this figure.

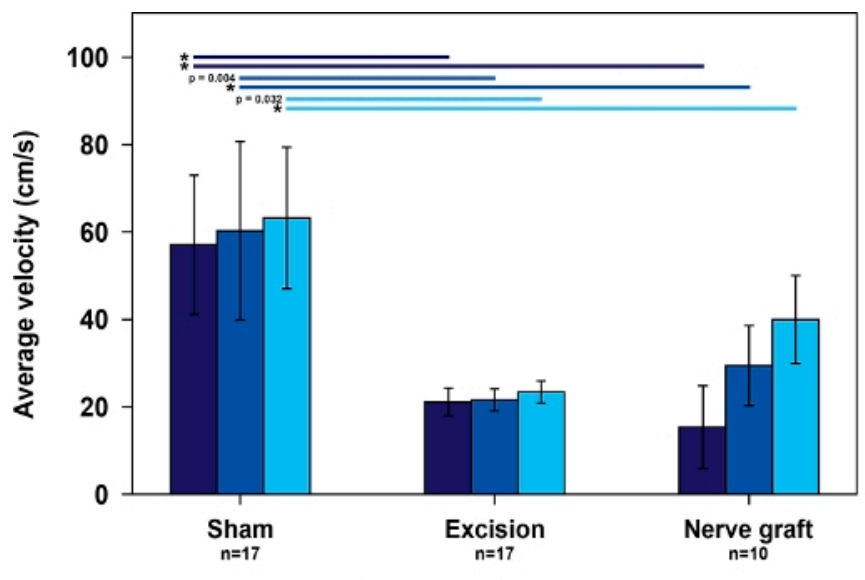

30 days after surgery

45 days after surgery

60 days after surgery

Experimental groups

Figure 5: Average speed in the ladder running test in the different experimental groups.

Vertical bars represent $95 \%$ confidence intervals. Asterisks in the upper portion of the figure indicate statistically significant differences between groups, ${ }^{*} p<0.001$. Please click here to view a larger version of this figure. 


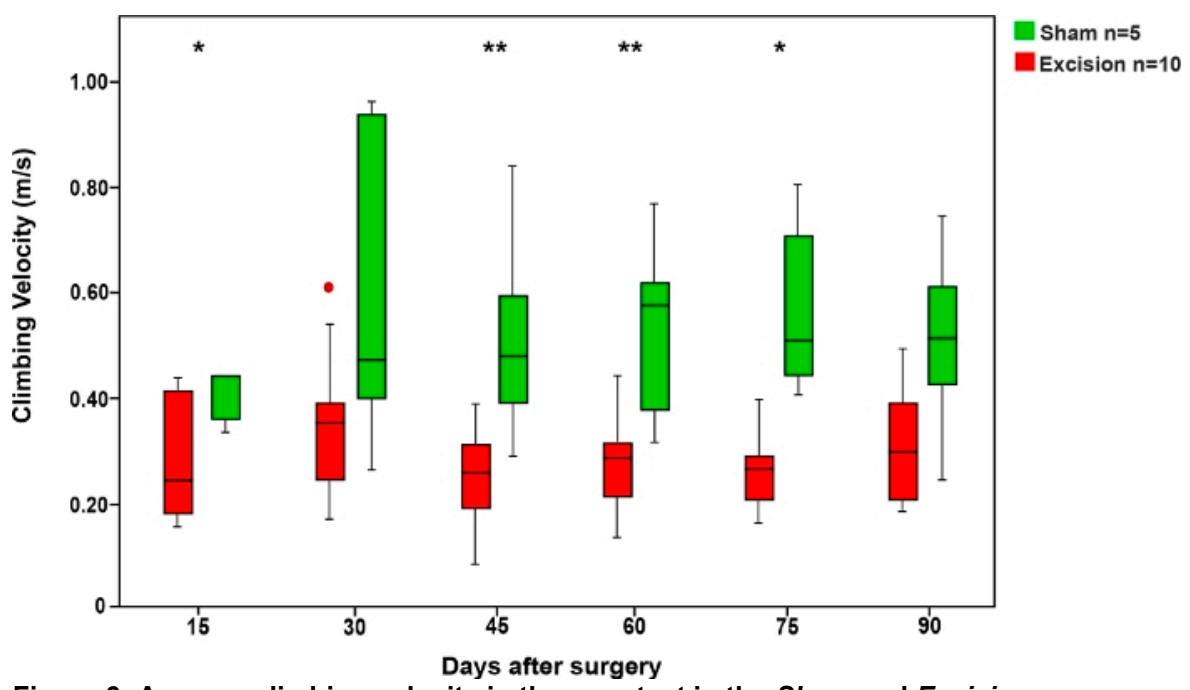

Figure 6: Average climbing velocity in the rope test in the Sham and Excision groups.

Vertical bars represent $95 \%$ confidence intervals. Asterisks in the upper portion of the figure show statistically significant differences between groups, ${ }^{*} p<0.05$; ${ }^{* *} p<0.01$. Please click here to view a larger version of this figure.
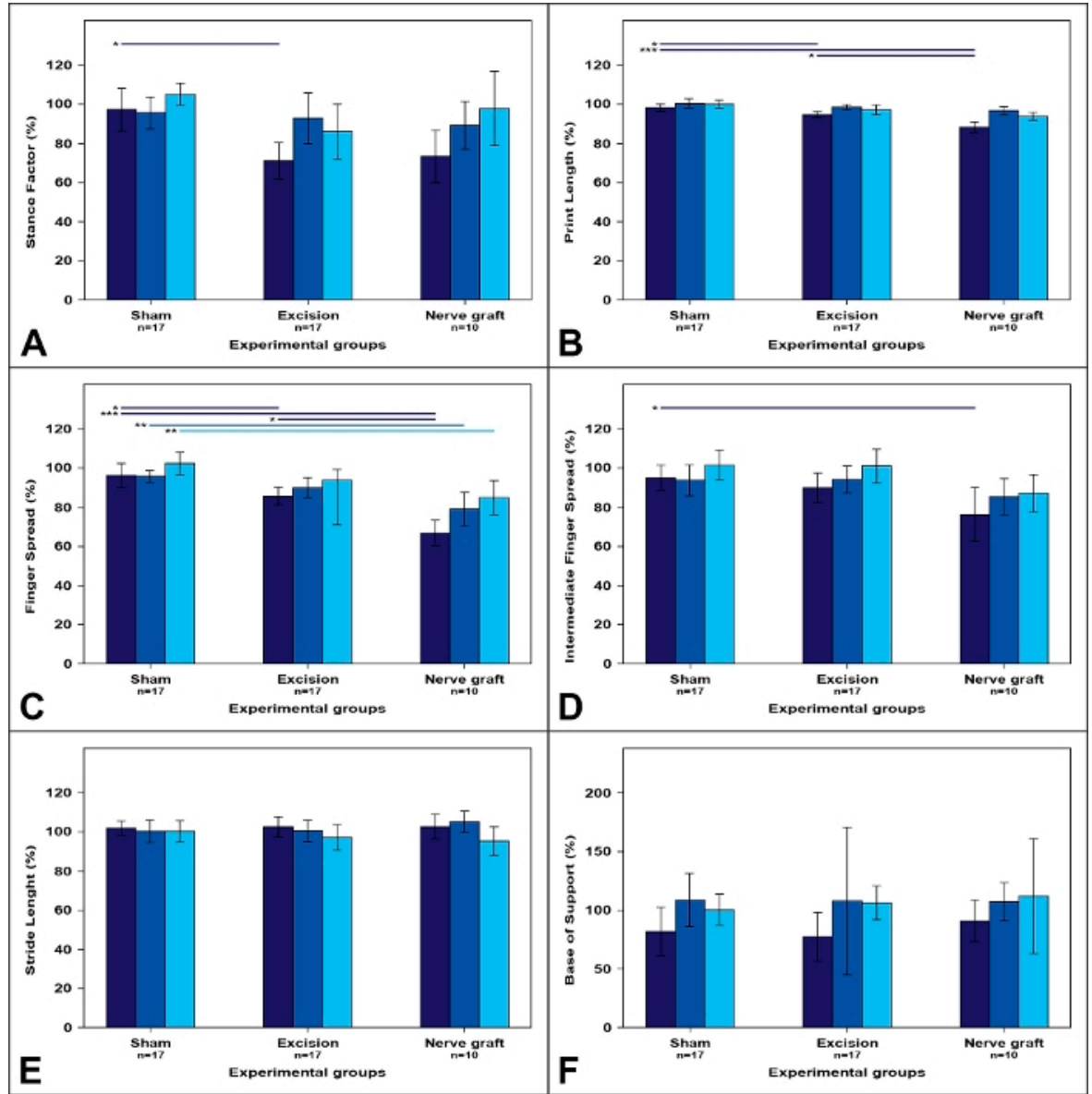

D30 $\square$ D60 $\square$ D90

Figure 7: Walking track parameters in the different experimental groups.

Values on the operated limb are expressed as percentages of means normalized to the contralateral limb. (A) Stance factor; (B) Print length; (C) Finger spread factor; (D) Intermediate finger spread factor; (E) Stride length; (F) Base of support. Vertical bars represent $95 \%$ confidence intervals. Horizontal lines in the upper portion of the figure indicate statistically significant differences between experimental groups. D30, D60, $\mathrm{D} 90=30,60$, and 90 days after surgery, ${ }^{*} \mathrm{p}<0.05 ;{ }^{* *} \mathrm{p}<0.01 ;{ }^{* * *} \mathrm{p}<0.001$. Please click here to view a larger version of this figure. 


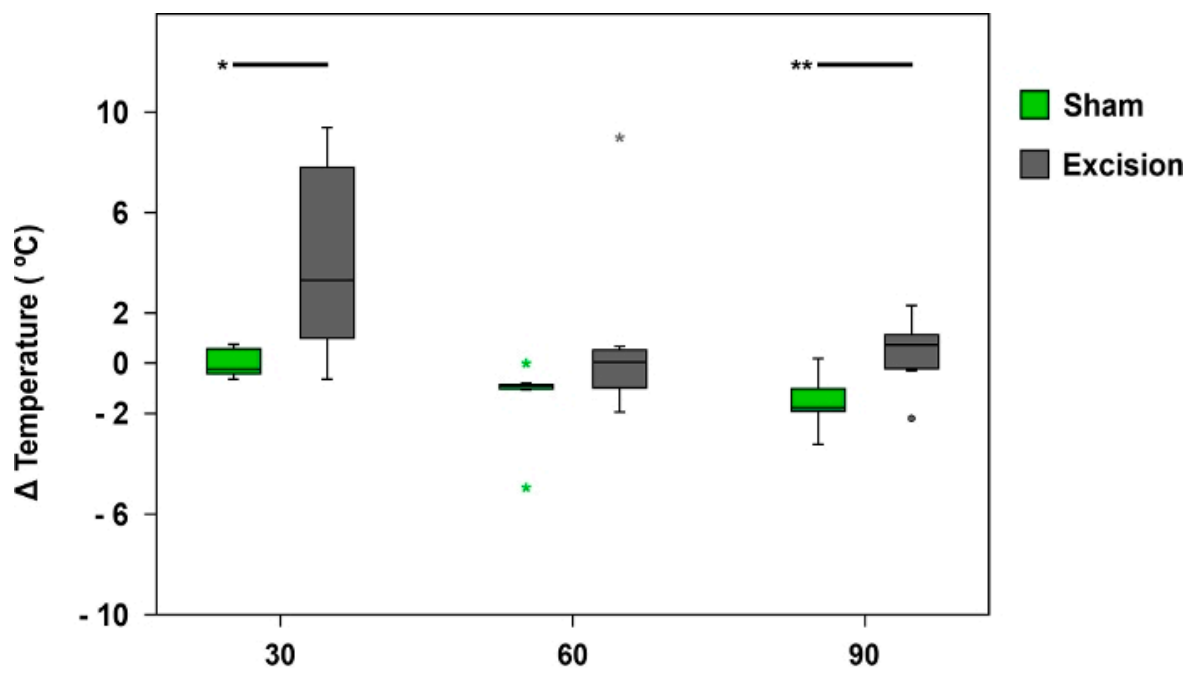

Days after surgery

Figure 8: Mean temperature difference registered by infrared thermography.

The box plots represent the temperature difference between the palmar region of the median nerve on the operated side (right side) and the contralateral side (left) in the Sham $(n=17)$ and Excision $(n=17)$ groups, ${ }^{*} p<0.05 ;{ }^{* *} p<0.01$. Please click here to view a larger version of this figure.
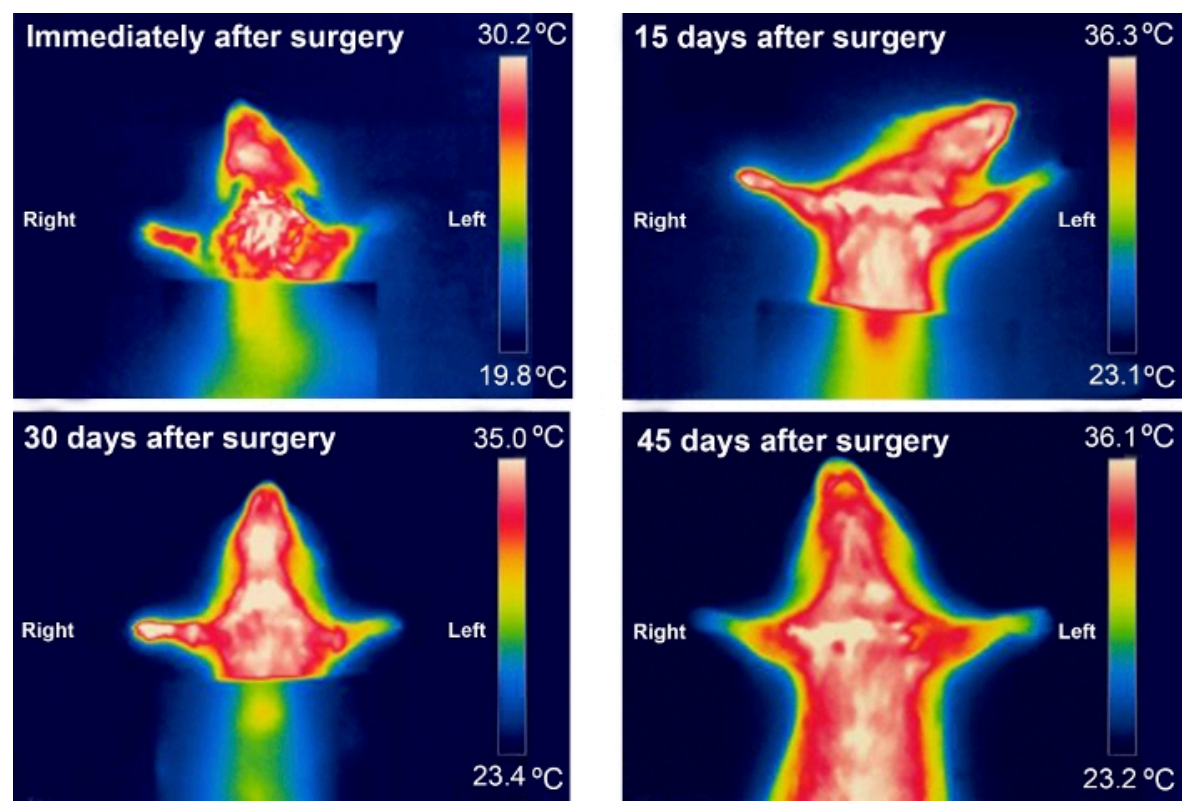

Figure 9: Typical infrared thermography pattern of an animal from the excision group during the first $\mathbf{4 5}$ days after surgery. Please click here to view a larger version of this figure. 
Normal CMAP pattern

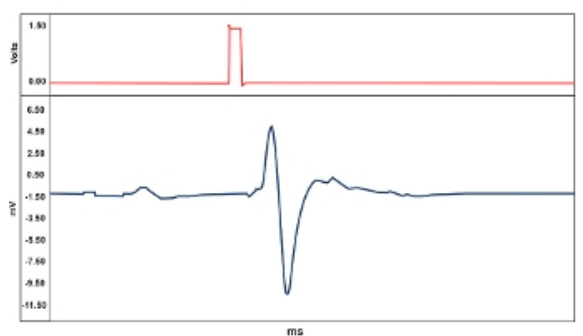

Sham Group

\section{Polyphasic CMAP pattern}

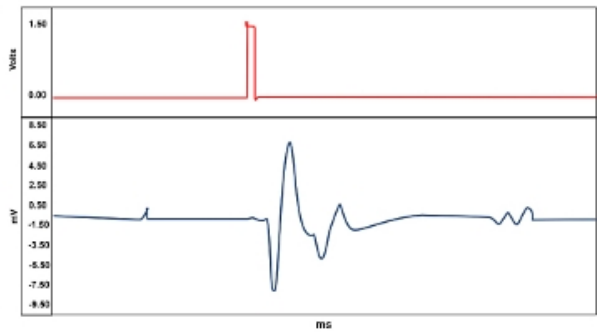

Nerve graft Group

Figure 10: Typical patterns of Compound Muscle Action Potentials (CMAPs) from an animal from the Sham and Nerve Graft groups 90 days after surgery. Please click here to view a larger version of this figure.

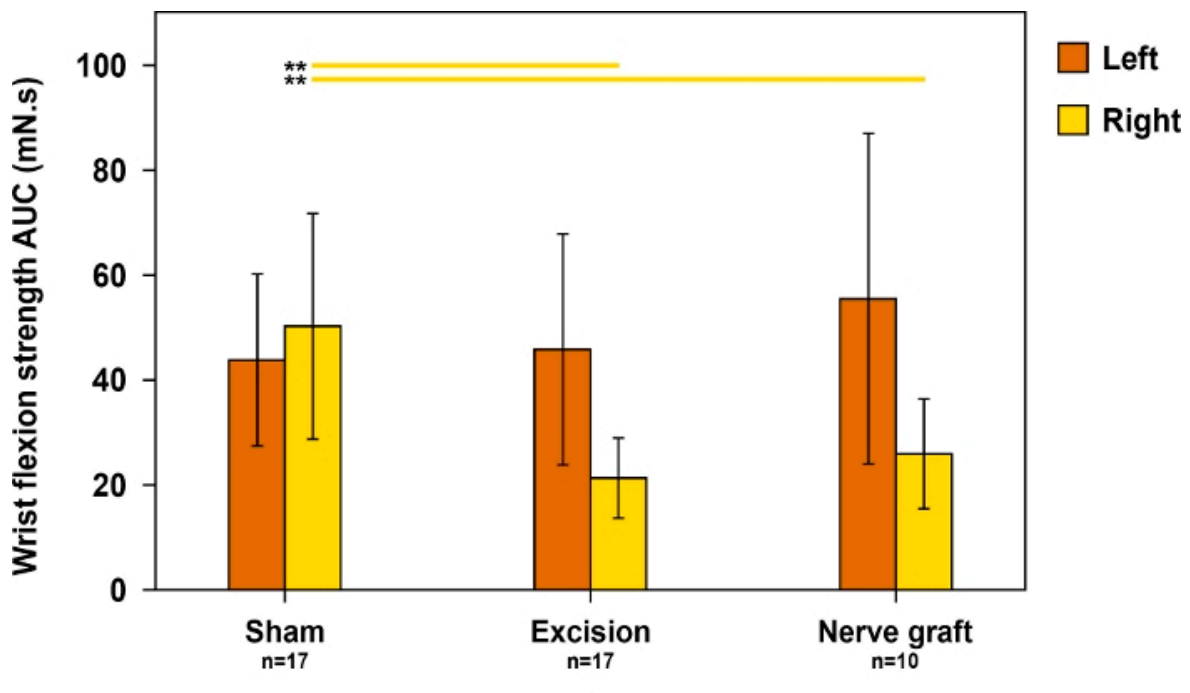

Experimental groups

Figure 11: Evaluation of wrist flexion strength on both forepaws 90 days postoperatively in different experimental groups.

Wrist flexion strength was assessed using the area under the curve (AUC) over a time period of $30 \mathrm{~s}$ and using supratetanic stimulation. Vertical lines denote $95 \%$ confidence intervals. Horizontal lines in the upper portion of the figure highlight statistically significant differences between groups, ${ }^{* *} p<0.01$. Please click here to view a larger version of this figure.

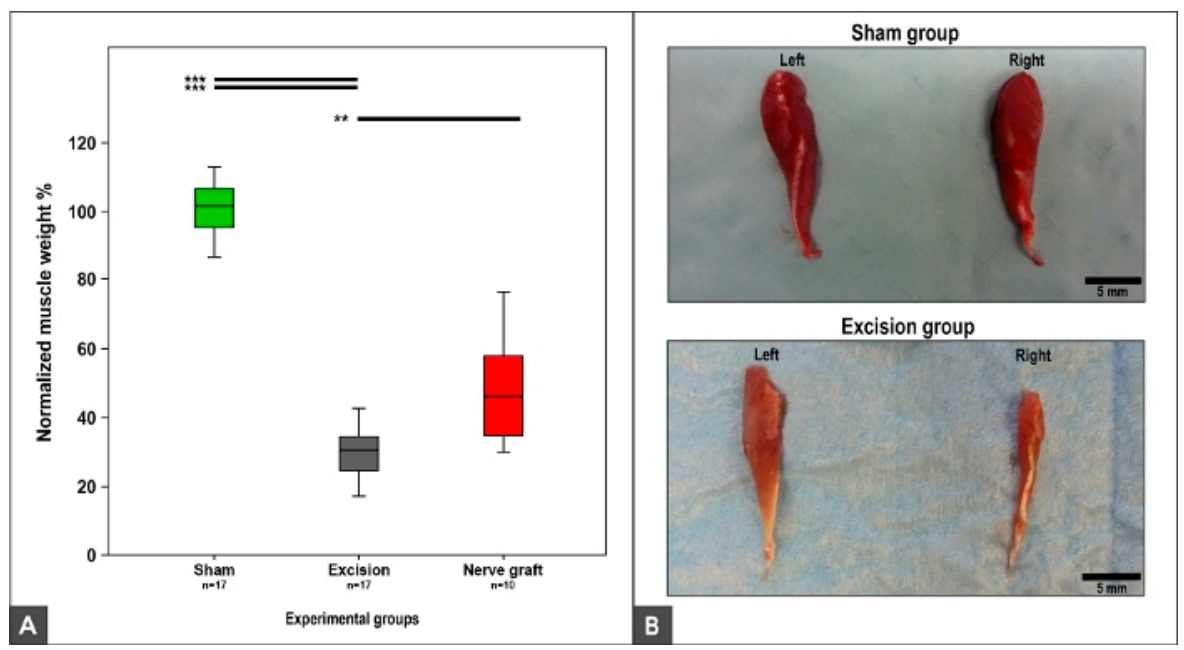

Figure 12: Flexor carpi radialis muscle weight and macroscopic appearance 100 days after surgery.

(A) Box plots depicting the normalized flexor carpi radialis muscle weight in different experimental groups, ${ }^{* *} p<0.01 ;{ }^{* * *} p<0.001$. (B) Photographs of the muscles on the right and left sides in the Sham and Excision experimental groups. Please click here to view a larger version of this figure. 


\begin{tabular}{|c|c|c|c|c|}
\hline Parameter & Parameter significance & Sham group & Excision group & NG group \\
\hline $\begin{array}{l}\text { Neurological stimulation } \\
\text { threshold (\%) }\end{array}$ & $\begin{array}{l}\text { Evaluation of nerve } \\
\text { regeneration, as there is a } \\
\text { minimal number of nerve } \\
\text { fibers required to produce } \\
\text { either a CMAP or a visible } \\
\text { muscle contraction }^{12}\end{array}$ & $281.63 \pm 271.65$ & $5359.98 \pm 3466.52$ & $2108.12 \pm 2115.13$ \\
\hline $\begin{array}{l}\text { Motor stimulation } \\
\text { threshold (\%) }\end{array}$ & $\begin{array}{l}\text { Evaluation of nerve } \\
\text { regeneration, as there is a } \\
\text { minimal number of nerve } \\
\text { fibers required to produce } \\
\text { either a CMAP or a visible } \\
\text { muscle contraction }^{12}\end{array}$ & $462.52 \pm 118.91$ & $1694.10 \pm 503.24$ & $1249.50 \pm 503.24$ \\
\hline Latency (\%) & $\begin{array}{l}\text { Assessment of nerve } \\
\text { conduction velocity in } \\
\text { the fastest nerve fibers, } \\
\text { that is to say the largest } \\
\text { myelinated fibers }\end{array}$ & $113.55 \pm 25.04$ & $\mathrm{~N} / \mathrm{A}$ & $132.80 \pm 69.95$ \\
\hline $\begin{array}{l}\text { Neuromuscular } \\
\text { transduction velocity (\%) }\end{array}$ & $\begin{array}{l}\text { Assessment of nerve } \\
\text { conduction velocity in } \\
\text { the fastest nerve fibers, } \\
\text { that is to say the largest } \\
\text { myelinated fibers }\end{array}$ & $92.01 \pm 20.88$ & $\mathrm{~N} / \mathrm{A}$ & $91.30 \pm 26.51$ \\
\hline CMAPs amplitude (\%) & $\begin{array}{l}\text { Evaluation of the number of } \\
\text { reinnervated motor units }\end{array}$ & $110.63 \pm 45.66$ & $\mathrm{~N} / \mathrm{A}$ & $41.60 \pm 24.84$ \\
\hline CMAPs duration (\%) & $\begin{array}{l}\text { Assessment of synchrony } \\
\text { of muscle innervation, } \\
\text { which is dependent on } \\
\text { the degree of muscle } \\
\text { reinnervation and } \\
\text { myelination of the } \\
\text { innervating motor fibers }\end{array}$ & $101.12 \pm 23.92$ & $\mathrm{~N} / \mathrm{A}$ & $151.06 \pm 54.52$ \\
\hline \multicolumn{5}{|c|}{$\begin{array}{l}\text { NG, nerve graft } \\
\text { CMAPs, compound muscle action potential. } \\
\text { N/A, non-applicable } \\
\text { All parameters are expressed as percentages of the average contralateral values. } \\
\text { Numeric variables are expressed as average } \pm \text { standard deviation. }\end{array}$} \\
\hline
\end{tabular}

Table 1: Electroneuromyographic assessment at the end of the experiment.

\section{Discussion}

This paper presents a protocol to create different types of MN lesions and repair in the rat. Additionally, it illustrates how to evaluate the functional recovery of this nerve using several noninvasive behavioral tests and physiological measurements.

Notably, several of the functional tests described in this paper, namely the Ladder Running Test and the Rope Test, are significantly dependent on the rat's willingness to perform the task with the expectation of obtaining the food reward $51,52,53$. It should be noted that certain rat strains are more amenable to training and performing reproducibly in this type of tests ${ }^{51,52,53}$. For example, Lewis rats perform poorly in these tests both in the training phase and subsequently ${ }^{51,52,53}$.

Rat housing should permit ample freedom of movement in agreement with their natural exploratory behavior, in addition to allowing experimental animals to get familiar with some of the elements present in the functional tests ${ }^{19}$. Therefore, different forms of housing allowing higher freedom of movement are shown. The big cages are personalized with enrichment elements that are later used in the functional tests (e.g., ropes and ladders).

Arguably, these enriching elements as well as the cages with incorporated running wheels and the individual training spheres provide a form of postoperative physiotherapy similar to that offered to human patients operated on the peripheral nervous $\operatorname{system}^{10}$.

Significantly, although some authors advocate dissecting the subcutaneous tissues and muscle fasciae bluntly or by clean cutting with a number 15 scalpel, the use of thermocautery when dissecting these structures is recommended to minimize the risk of postoperative hematoma.

It should be noted that numerous tests have been devised to test different aspects of peripheral nerve repair in the rat, namely axonal regeneration, target reinnervation, and functional recovery, some of which are beyond the scope of this study ${ }^{29,54,55,56}$. For example, kinematic analysis $^{29,36,55}$ and histomorphometric assessment ${ }^{29,36,57}$ are widely employed by multiple authors. Additionally, several of these tests involve 
variations to maximize efficiency and/or reproducibility ${ }^{54}$. For example, mechanical algisemetry (i.e., evaluation of responses to mechanical painful stimuli) can be assessed qualitatively using a given von Frey filament, as described in the present paper, or semiquantitatively using successively stronger von Frey filaments, or even quantitatively using electronic devices that apply increasing pressures until a withdrawal response is observed ${ }^{30,54}$

Similarly, although several authors use walking track analysis to evaluate forelimb nerve repair in the rat, other authors argue that single $\mathrm{MN}$ lesions frequently fail to produce reproducible changes in pawprints ${ }^{10,58,59}$. Furthermore, some have stated that these changes may not be proportional to muscle recovery ${ }^{10,60}$. Bearing this in mind, some researchers have advocated the use of walking track analysis in the forepaw mainly when assessing recovery after crushing neve lesions rather than after segmental nerve reconstruction ${ }^{10,50,61}$.

The Grasping Test is widely used to evaluate motor recovery of the muscles controlled by the $\mathrm{MN}^{16,27}$. To guarantee uniformity and reproducibility of the data obtained with this test, applying the Grasping Test using the well-established methodology proposed by Bertelli et al. ${ }^{16}$ is recommended. However, the current protocol differs in that it does not routinely immobilize the contralateral paw to prevent undue stress ${ }^{11,27}$. It should also be noted that other authors, after immobilizing the uninjured paw, quantitatively assess the Grasping Test using a dynamometer or a scale $e^{27,56}$. However, this quantitative evaluation may be affected by the strength the researcher applies to the rat's tail ${ }^{26}$. Furthermore, it is difficult to distinguish between the strength generated by the digital flexor muscles (solely innervated by the MN in the rat and the object of the Grasping Test ${ }^{9}$ ) from the strength produced by the wrist flexors, which include the flexor carpi ulnaris that receives its innervation from the ulnar nerve $e^{9,10,27}$. In order to try to circumvent these potential biases, this protocol uses an ordinal scale similar to the Medical Research Council Scale commonly used to grade muscle strength in humans ${ }^{10,11,62}$. Alternatively, other authors have described detailed assessment of grasping using video analysis and a video-based scoring system ${ }^{11,63}$.

A potential disadvantage of using the $\mathrm{MN}$ compared to the sciatic nerve is that a greater amount of information is available regarding the latter nerve. This, in turn, can make comparison of data obtained with the MN with that of prior experimental works more difficult ${ }^{46,48,64}$. Additionally, the smaller size of the MN compared to the sciatic nerve makes surgical manipulation more challenging $8,12,27,56,65$.

Contrary to the methodology described in this paper, the electroneuromyography evaluation can be performed using transcutaneous monopolar electrodes placed in the arm and thenar regions ${ }^{51}$. Despite being less invasive, this method carries the risk of potential confusion due to the possibility of costimulation of the ulnar nerve in the arm region ${ }^{9,51}$.

Most authors concur that not all tests used in the rat provide concordant results, as peripheral nerve repair depends on a complex array of factors, comprising neuron survival, axonal elongation and pruning, synaptogenesis, successful recapture of the denervated sensory organs and motor units, and brain plasticity $7,10,50,66,67$

Finally, it should be noted that a significant caveat of rodent models is that rat peripheral nerves are much closer to their end organs and have much smaller cross-sectional areas than the homologous human structures. However, this size difference guarantees faster experimental data in rodents, and better overall results in rats in comparison to humans are to be expected ${ }^{68}$. Indeed, several authors warn that care must be used when trying to extrapolate experimental data obtained in peripheral nerve repair using rodents to humans ${ }^{7,69}$. Primate models are considered more comparable ${ }^{70}$. Nevertheless, their use is associated with vexing ethical, logistical, and budget constraints ${ }^{71}$.

Even though the sciatic nerve is the most commonly used nerve in peripheral nerve research, the rat MN presents multiple advantages. For example, MN lesions are associated with a smaller incidence of joint contractures and automutilation of the affected paw ${ }^{11,12,16,56}$. Significantly, autotomy subsequent to sciatic nerve transection afflicts $11-70 \%$ of rats. This may make current evaluations like the sciatic index impossible ${ }^{14}$. This, in turn, makes the estimate of the number of animals required to obtain a given statistical power cumbersome ${ }^{15}$

In addition, as the $\mathrm{MN}$ is shorter than the sciatic nerve, nerve recovery is observed sooner ${ }^{58,72,73,74,75,76}$. Furthermore, the MN is not covered by muscle masses, making its dissection technically easier than that of the sciatic nerve ${ }^{16}$. Additionally, the MN has a parallel path to the ulnar nerve in the arm. Hence, the ulnar nerve can easily be used as nerve graft for repairing MN injuries. Finally, in humans, most peripheral nerve lesions occur in the upper limb, which further supports the use of this nerve in the rat ${ }^{77,78}$.

Arguably, rodents are the experimental animals most commonly used in the realm of peripheral nerve repair ${ }^{48,79}$. As shown, the rat MN is a convenient model of peripheral nerve lesion and repair. In fact, there are multiple standardized strategies available to assess motor and sensory recovery, permitting an easier comparison of results ${ }^{36,46,60,80,81,82}$. Many of these methods are noninvasive, allowing for daily assessment.

Moreover, physiotherapy is part of the standard of care of patients recovering from peripheral nerve injuries. As demonstrated in this paper, there are multiple strategies to provide a postoperative physiotherapy-like environment to rats submitted to MN injuries, ${ }^{4,5}$. Hence, this model is particularly suitable to replicate the clinical scenario, facilitating extrapolation of results to the human species ${ }^{12,27,48,56,58,83}$.

As shown in this paper, multiple standardized strategies are available to assess motor and sensory recovery in the MN model of the rat. The majority of these are noninvasive procedures, allowing frequent assessment. Moreover, as most peripheral nerve lesions in the human species occur in the upper limb, the mentioned experimental physiotherapy settings can more aptly mimic recovery in the clinical context. Arguably, this can facilitate extrapolation of results to the human species, further validating the use of this nerve in the rat.

\section{Disclosures}

The authors have nothing to disclose.

\section{Acknowledgments}

Diogo Casal received a grant from The Program for Advanced Medical Education, which is sponsored by Fundação Calouste Gulbenkian, Fundação Champalimaud, Ministério da Saúde e Fundação para a Ciência e Tecnologia, Portugal. The authors are very grateful to Mr. Filipe 
Franco for the illustrative drawing in Figure 1. The authors would like to thank the technical help of Mr. Alberto Severino in filming and editing the video. Finally, the authors would like to thank Ms. Sara Marques for her help in all the logistical aspects pertaining to animal acquisition and maintenance.

\section{References}

1. Lad, S. P., Nathan, J. K., Schubert, R. D., Boakye, M. Trends in median, ulnar, radial, and brachioplexus nerve injuries in the United States. Neurosurgery. 66 (5), 953-960 (2010).

2. Murovic, J. A. Upper-extremity peripheral nerve injuries: a Louisiana State University Health Sciences Center literature review with comparison of the operative outcomes of 1837 Louisiana State University Health Sciences Center median, radial, and ulnar nerve lesions. Neurosurgery. 65 (4 Suppl), A11-17 (2009).

3. Dy, C. J., Isaacs, J. in American Society for Surgery of the Hand surgical anatomy: nerve reconstruction. Vol. 1 eds. C. J. Dy and J. Isaacs) xi American Society for Surgery of the Hand, (2017).

4. Trehan, S. K., Model, Z., Lee, S. K. Nerve Repair and Nerve Grafting. Hand Clinics. 32 (2), 119-125 (2016).

5. Boyd, K. U., Fox, I. K. in Nerve surgery. Vol. 1 (ed. S. E. Mackinnon) Ch. 4, pp. 75-100 Thieme, (2015).

6. Geuna, S. et al. Update on nerve repair by biological tubulization. Journal of Brachial Plexius Peripheral Nerve Injury. 9 (1), 3 (2014).

7. Sulaiman, W., Gordon, T. Neurobiology of peripheral nerve injury, regeneration, and functional recovery: from bench top research to bedside application. Ochsner Journal. 13 (1), 100-108 (2013).

8. Angelica-Almeida, M. et al. Brachial plexus morphology and vascular supply in the wistar rat. Acta Medica Portuguesa. 26 (3), $243-250$ (2013).

9. Bertelli, J. A., Taleb, M., Saadi, A., Mira, J. C., Pecot-Dechavassine, M. The rat brachial plexus and its terminal branches: an experimental model for the study of peripheral nerve regeneration. Microsurgery. 16 (2), 77-85 (1995).

10. Casal, D. et al. Reconstruction of a 10-mm-long median nerve gap in an ischemic environment using autologous conduits with different patterns of blood supply: A comparative study in the rat. PLoS One. 13 (4), e0195692 (2018).

11. Stößel, M., Rehra, L., Haastert-Talini, K. Reflex-based grasping, skilled forelimb reaching, and electrodiagnostic evaluation for comprehensive analysis of functional recovery-The 7-mm rat median nerve gap repair model revisited. Brain and Behavior. 7 (10), e00813 (2017).

12. Manoli, T. et al. Correlation analysis of histomorphometry and motor neurography in the median nerve rat model. Eplasty. 14, e17 (2014).

13. Ronchi, G. et al. The Median Nerve Injury Model in Pre-clinical Research - A Critical Review on Benefits and Limitations. Frontiers in Cellular Neuroscience. 13, 288 (2019).

14. Weber, R. A., Proctor, W. H., Warner, M. R., Verheyden, C. N. Autotomy and the sciatic functional index. Microsurgery. 14 (5), $323-327$ (1993).

15. Charan, J., Kantharia, N. D. How to calculate sample size in animal studies? Journal of Pharmacology \& Pharmacotherapeutics. 4 (4), 303-306 (2013).

16. Bertelli, J. A., Mira, J. C. The grasping test: a simple behavioral method for objective quantitative assessment of peripheral nerve regeneration in the rat. Journal of Neuroscience Methods. 58 (1-2), 151-155 (1995).

17. Casal, D. et al. A Model of Free Tissue Transfer: The Rat Epigastric Free Flap. Journal of Visualized Experiments. (119), e55281 (2017).

18. Bertens, A. P. M. G. et al. Anaesthesia, analgesia and euthanasia. In: Principles of Laboratory Animal Science. Eds. Van Zuthphen, L. F., Baumans, V., Beynen, A.C. Elsevier. pp. 277-311 (2001).

19. Pritchett-Corning, K. R., Luo, Y., Mulder, G. B., White, W. J. Principles of rodent surgery for the new surgeon. Journal of Visualized Experiments. (47), e2586 (2011).

20. Lee-Parritz, D. Analgesia for rodent experimental surgery. Israel Journal of Veterinary Medicine. 62 (3), 74 (2007).

21. Roughan, J. V., Flecknell, P. A. Evaluation of a short duration behaviour-based post-operative pain scoring system in rats. European Journal of Pain. 7 (5), 397-406 (2003).

22. Bauder, A. R., Ferguson, T. A. Reproducible mouse sciatic nerve crush and subsequent assessment of regeneration by whole mount muscle analysis. Journal of Visualized Experiments. (60), e3606 (2012).

23. Ronchi, G. et al. Functional and morphological assessment of a standardized crush injury of the rat median nerve. Journal of Neuroscience Methods. 179 (1), 51-57 (2009).

24. Matsumine, H. et al. Vascularized versus nonvascularized island median nerve grafts in the facial nerve regeneration and functional recovery of rats for facial nerve reconstruction study. Journal of Reconstructive Microsurgery. 30 (2), 127-136 (2014).

25. Mickley, A. G., Hoxha, Z., Biada, J. M., Kenmuir, C. L., Bacik, S. E. Acetaminophen Self-administered in the Drinking Water Increases the Pain Threshold of Rats (Rattus norvegicus). Journal of the American Association for Laboratory Animal Science. 45 (5), $48-54$ (2006).

26. Mandillo, S. et al. Reliability, robustness, and reproducibility in mouse behavioral phenotyping: a cross-laboratory study. Physiological Genomics. 34 (3), 243-255 (2008).

27. Papalia, I., Tos, P., Stagno d'Alcontres, F., Battiston, B., Geuna, S. On the use of the grasping test in the rat median nerve model: a reappraisal of its efficacy for quantitative assessment of motor function recovery. Journal of Neuroscience Methods. 127 (1), $43-47$ (2003).

28. Costa, L. M., Simoes, M. J., Mauricio, A. C., Varejao, A. S. Chapter 7: Methods and protocols in peripheral nerve regeneration experimental research: part IV-kinematic gait analysis to quantify peripheral nerve regeneration in the rat. International Reviews in Neurobiology. 87, 127-139 (2009).

29. Geuna, S., Varejao, A. S. Evaluation methods in the assessment of peripheral nerve regeneration. Journal of Neurosurgery. 109 (2), $360-362$; author reply 362, (2008).

30. Howard, R. F., Hatch, D. J., Cole, T. J. Fitzgerald, M. Inflammatory pain and hypersensitivity are selectively reversed by epidural bupivacaine and are developmentally regulated. Anesthesiology. 95 (2), 421-427 (2001).

31. Metz, G. A., Whishaw, I. Q. Cortical and subcortical lesions impair skilled walking in the ladder rung walking test: a new task to evaluate foreand hindlimb stepping, placing, and co-ordination. Journal of Neuroscience Methods. 115 (2), 169-179 (2002).

32. Thallmair, M. et al. Neurite growth inhibitors restrict plasticity and functional recovery following corticospinal tract lesions. Nature Neuroscience. 1 (2), 124-131 (1998).

33. Brown, C. J. et al. Self-evaluation of walking-track measurement using a Sciatic Function Index. Microsurgery. 10 (3), $226-235$ (1989).

34. Hruska, R. E., Kennedy, S., Silbergeld, E. K. Quantitative aspects of normal locomotion in rats. Life Science. 25 (2), 171-179 (1979). 
35. Ferreira, T., Rasband, W. ImageJ user guide. ImageJ/Fiji. 1, 155-161 (2012).

36. Dijkstra, J. R., Meek, M. F., Robinson, P. H., Gramsbergen, A. Methods to evaluate functional nerve recovery in adult rats: walking track analysis, video analysis and the withdrawal reflex. Journal of Neuroscience Methods. 96 (2), 89-96 (2000).

37. Ludwig, N., Formenti, D., Gargano, M., Alberti, G. Skin temperature evaluation by infrared thermography: Comparison of image analysis methods. Infrared Physics \& Technology. 62, 1-6 (2014).

38. Bennett, G. J., Ochoa, J. L. Thermographic observations on rats with experimental neuropathic pain. Pain. 45 (1), $61-67$ (1991).

39. Wakisaka, S., Kajander, K. C., Bennett, G. J. Abnormal skin temperature and abnormal sympathetic vasomotor innervation in an experimental painful peripheral neuropathy. Pain. 46 (3), 299-313 (1991).

40. Muntean, M. V. et al. Using dynamic infrared thermography to optimize color Doppler ultrasound mapping of cutaneous perforators. Medical Ultrasonography. 17 (4), 503-508 (2015).

41. Shterenshis, M. Challenges to Global Implementation of Infrared Thermography Technology: Current Perspective. Central Asian Journal of Global Health. 6 (1), 289 (2017).

42. Wilbourn, A. J. in Nerve surgery. Vol. 1 (ed. S. E. Mackinnon) Ch. 3, pp. 59-74 Thieme, (2015).

43. Wu, Y., Martínez, M. Á. M., Balaguer, P. O. in Electrodiagnosis in New Frontiers of Clinical Research. (ed. Hande Turker) Ch. 01 InTech, (2013).

44. Werdin, F. et al. An improved electrophysiological method to study peripheral nerve regeneration in rats. Journal of Neuroscience Methods. 182 (1), 71-77 (2009)

45. Sedghamiz, H., Santonocito, D. Unsupervised Detection and Classification of Motor Unit Action Potentials in Intramuscular Electromyography Signals. 2015 E-health and Bioengineering Conference IEEE. pp. 1-6. (2015).

46. Hadlock, T. A., Koka, R., Vacanti, J. P., Cheney, M. L. A comparison of assessments of functional recovery in the rat. Journal of the Peripheral Nervous System. 4 (3-4), 258-264 (1999).

47. Carstens, E., Moberg, G. P. Recognizing pain and distress in laboratory animals. Ilar Journal. 41 (2), 62-71 (2000).

48. Tos, P. et al. Chapter 4: Methods and protocols in peripheral nerve regeneration experimental research: part l-experimental models. International Reviews in Neurobiology. 87, 47-79 (2009).

49. Galtrey, C. M., Fawcett, J. W. Characterization of tests of functional recovery after median and ulnar nerve injury and repair in the rat forelimb. Journal of the Peripheral Nervous System. 12 (1), 11-27 (2007).

50. Giusti, G. et al. Return of motor function after segmental nerve loss in a rat model: comparison of autogenous nerve graft, collagen conduit, and processed allograft (AxoGen). Journal of Bone and Joint Surgery American Volume. 94 (5), 410-417 (2012).

51. Stossel, M., Rehra, L., Haastert-Talini, K. Reflex-based grasping, skilled forelimb reaching, and electrodiagnostic evaluation for comprehensive analysis of functional recovery-The 7-mm rat median nerve gap repair model revisited. Brain and Behavior. 7 (10), e00813 (2017).

52. Nikkhah, G., Rosenthal, C., Hedrich, H. J., Samii, M. Differences in acquisition and full performance in skilled forelimb use as measured by the 'staircase test' in five rat strains. Behavioral Brain Research. 92 (1), 85-95 (1998).

53. Whishaw, I. Q., Gorny, B., Foroud, A., Kleim, J. A. Long-Evans and Sprague-Dawley rats have similar skilled reaching success and limb representations in motor cortex but different movements: some cautionary insights into the selection of rat strains for neurobiological motor research. Behavioral Brain Research. 145 (1-2), 221-232 (2003).

54. Navarro, X. Functional evaluation of peripheral nerve regeneration and target reinnervation in animal models: a critical overview. European Journal of Neuroscience. 43 (3), 271-286 (2016).

55. Costa, L. M., Simões, M. J., Maurício, A. C., Varejão, A. S. P. in International Review of Neurobiology. Vol. 87, pp. 127-139 Academic Press, (2009).

56. Ronchi, G. et al. Functional and morphological assessment of a standardized crush injury of the rat median nerve. Journal of Neuroscience Methods. 179 (1), 51-57 (2009).

57. Raimondo, S. et al. Chapter 5: Methods and protocols in peripheral nerve regeneration experimental research: part II-morphological techniques. International Reviews in Neurobiology. 87, 81-103 (2009).

58. Bontioti, E. K. M., Dahlin L. B. Regeneration and functional recovery in the upper extermity of rats after various types of nerve injuries. Journal of the Peripheral Nervous System. 8, 159-168 (2003).

59. Schönfeld, L.M., Dooley, D., Jahanshahi, A., Temel, Y., Hendrix, S. Evaluating rodent motor functions: Which tests to choose? Neuroscience \& Biobehavioral Reviews. 83, 298-312 (2017).

60. Urbancheck, M. S. Rat walking tracks do not reflect maximal muscle force capacity. Journal of Reconstructive Microsurgery. 15 (2), $143-149$ (1999).

61. Cudlip, S. A., Howe, F. A., Griffiths, J. R., Bell, B. A. Magnetic resonance neurography of peripheral nerve following experimental crush injury, and correlation with functional deficit. Journal of Neurosurgery. 96 (4), 755-759 (2002).

62. Wang, Y., Sunitha, M., Chung, K. C. How to measure outcomes of peripheral nerve surgery. Hand Clinics. 29 (3), $349-361$ (2013).

63. Wang, H., Spinner, R. J., Sorenson, E. J., Windebank, A. J. Measurement of forelimb function by digital video motion analysis in rat nerve transection models. Journal of the Peripheral Nervous System. 13 (1), 92-102 (2008).

64. Yanase, Y. in Experimental and Clinical Reconstructive Microsurgery. eds S. Tamai, M. Usui, T. Yoshizu) Ch. 2, pp. 44-51 Springer-Verlag, (2004).

65. Barton, M. J. et al. Morphological and morphometric analysis of the distal branches of the rat brachial plexus. Italian Journal of Anatomy and Embryology. 121 (3), 240-252 (2016).

66. Vincent, R. Adult and obstetrical brachial plexus injuries. In: Peripheral Nerve Surgery: Practical applications in the upper extremity. Eds. Slutsky D. J., Hentz, V. R. Churchill Livingstone. pp. 299-317 (2006).

67. Dahlin, L. B. in Peripheral Nerve Surgery: Practical Applications in the Upper Extremity. eds. Slutsky, D. J., Hentz, V. R.) Ch. 1, pp. 1-22 Elsevier, (2006).

68. Vargel, I. et al. A comparison of various vascularization-perfusion venous nerve grafts with conventional nerve grafts in rats. Journal of Reconstructive Microsurgery. 25 (7), 425-437 (2009).

69. Grinsell, D., Keating, C. Peripheral nerve reconstruction after injury: a review of clinical and experimental therapies. BioMed Research International. 2014, 698256 (2014).

70. Wang, D. et al. A simple model of radial nerve injury in the rhesus monkey to evaluate peripheral nerve repair. Neural Regeneration Research. 9 (10), 1041-1046 (2014). 
71. Casal, D. et al. Unconventional Perfusion Flaps in the Experimental Setting: A Systematic Review and Meta-Analysis. Plastic Reconstructive Surgery. 143 (5), 1003e-1016e (2019).

72. Bontioti, E. End-to-side nerve repair. A study in the forelimb of the rat. PhD thesis, Lund, (2005).

73. Bodine-Fowler, S. C., Meyer, R. S., Moskovitz, A., Abrams, R. Botte, M. J. Inaccurate projection of rat soleus motoneurons: a comparison of nerve repair techniques. Muscle Nerve. 20 (1), 29-37 (1997)

74. Valero-Cabre, A., Navarro, X. H reflex restitution and facilitation after different types of peripheral nerve injury and repair. Brain Research. 919 (2), 302-312 (2001).

75. Wall, P. D. et al. Autotomy following peripheral nerve lesions: experimental anaesthesia dolorosa. Pain. 7 (2), $103-111$ (1979).

76. Bertelli, J. A., Taleb, M., Saadi, A., Mira, J. C., Pecot-Dechavassine, M. The rat brachial plexus and its terminal branches: an experimental model for the study of peripheral nerve regeneration. Microsurgery. 16, 77-85 (1995).

77. Wood, M. J., Johnson, P. J., Myckatyn, T. M. in Nerve Surgery. Vol. 1 eds. Mackinnon, S. E., Yee, A.) Ch. 1, pp. 1-40 Thieme, (2015).

78. Rosberg, H. E. Epidemiology of hand injuries in a middle-sized city in southern Sweden - a retrospective study with an 8-year interval. Scandinavian Journal of Plastic and Reconstructive Surgery and Hand Surgery. 38, 347-355 (2004).

79. Gordon, T., Borschel, G. H. The use of the rat as a model for studying peripheral nerve regeneration and sprouting after complete and partial nerve injuries. Experimental Neurology. 287 (Pt 3), 331-347 (2017).

80. Bertelli, J. A., Ghizoni, M. F. Concepts of nerve regeneration and repair applied to brachial plexus reconstruction. Microsurgery. 26 (4), 230-244 (2006).

81. Bertelli, J. A., Mira, J. C. Behavioural evaluating methods in the objective clinical assessment of motor function after experimental brachial plexus reconstruction in the rat. Journal of Neuroscience Methods. 46, 203-208 (1993).

82. Bertelli, J. A., Mira, J. C. The grasping test: a simple behavioral method for objective quantitative assessment of peripherla nerve regeneration in the rat. Journal of Neuroscience Methods. 58 (1-2), 151-155 (1995).

83. Ronchi, G. et al. Standardized crush injury of the mouse median nerve. Journal of Neuroscience Methods. 188 (1), 71-75 (2010). 